\title{
Heartbeat: weight loss interventions in patients with cardiovascular disease
}

Overweight and obesity are major risk factors for cardiovascular disease (CVD) and meaningful weight loss is associated with a reduction in CVD risk. Yet patients' efforts at weight reduction often are frustratingly futile. In this issue of Heart, Tijssen and colleagues ${ }^{1}$ evaluated weight change from baseline to 12-month follow-up in the subgroup of overweight patients (BMI $\geq 27 \mathrm{~kg} / \mathrm{m}^{2}$ ) with coronary artery disease in the Randomised Evaluation of Secondary Prevention by Outpatient Nurse SpEcialists-2 (RESPONSE-2) multicentre randomised trial. The 280 patients in the intervention arm were offered community-based programmes to achieve weight reduction, increase physical activity and stop smoking in addition usual care, which included cardiology visits, cardiac rehabilitation and counselling on secondary prevention. Although there was wide variation in weight loss for patients in both the intervention and usual care groups, participation in a weight loss programme was associated with weight loss of $\geq 5 \%$ (OR 3.33 compared with usual care) (figure 1). Other factors associated with meaningful weight loss were older age, lower educational level, not smoking and motivation to start weight loss at the baseline visit.

This data supports the wider use of community-based weight loss programmes as a feasible and low cost approach to meaningful weight reduction in our patients with CVD. However, as Kelsey and Pagidipati comment in an editorial" "Beyond behavioural and psychosocial characteristics, molecular and genetic factors also play a role in response to weight loss interventions" (figure 2). They argue that "Development of a personalised approach can and should match patient to treatment to obtain maximally effective and durable weight loss results."

Both coronary artery (CAC) and aortic valve calcification (AVC) are associated with an increased risk of CVD events but it remains unclear whether this simply

Correspondence to Professor Catherine M Otto, Division of Cardiology, University of Washington, Seattle,WA 98195, USA; cmotto@uw.edu
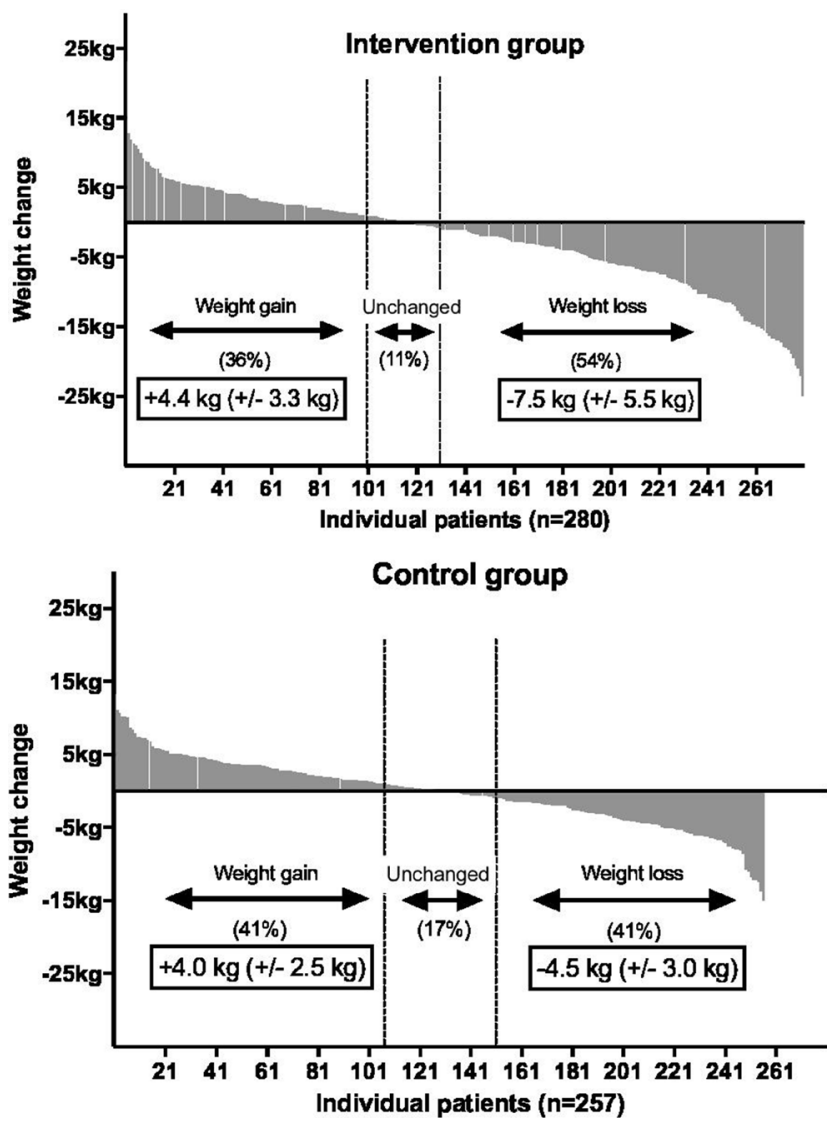

Figure 1 Waterfall plots; weight change from baseline to 12-month follow-up stratified by randomised treatment allocation.

reflects shared clinical risk factors or whether AVC scores provide additive predictive value, independent of CAC score, for CVD events. ${ }^{3}$ In a study of 14073 men aged 60 to 74 years of age in the DANish CArdioVAscular Screening and intervention trial (DANCAVAS), AVC

was present in $58 \%$ with $1.5 \%$ having an AVC score $\geq 1200$ (suggestive of severe aortic stenosis). After accounting for CAC scores, the only CVD risk factors independently associated with AVC were older age, hypertension, obesity, known CVD and serum phosphate levels.
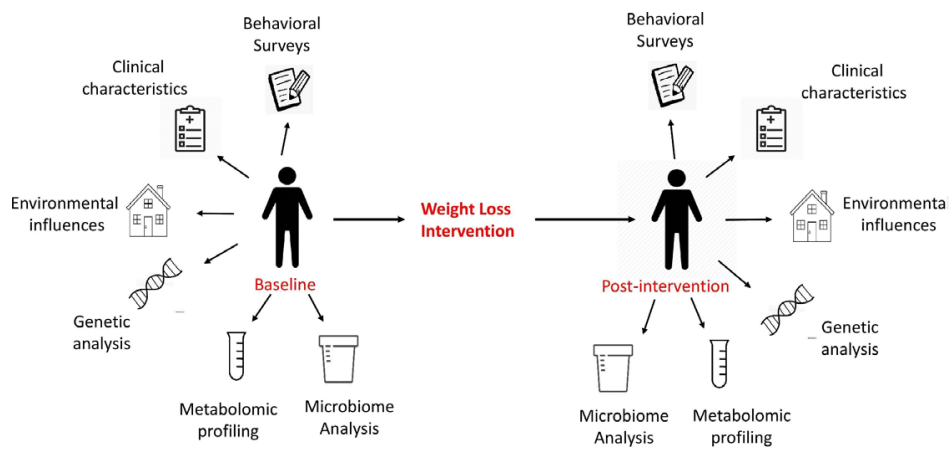

Figure 2 Proposed phenotyping of individuals before and after weight loss interventions. 


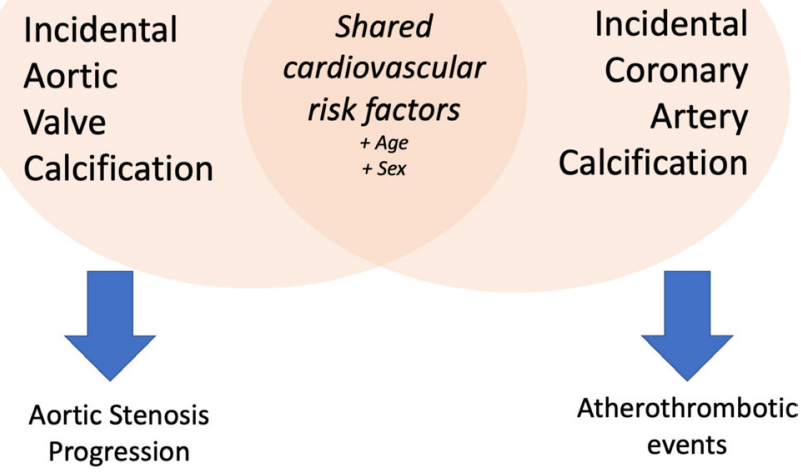

Figure 3 Aortic valve calcification and coronary artery calcification share overlapping risk factors, but whether this is due to association or causation remains uncertain.

Williams and Dweck $^{4}$ point out that "90\% of patients with aortic valve calcification also had coronary artery calcification, and $62 \%$ of patients with coronary artery calcification also had aortic valve calcification." Putting these findings in context of previous studies, we also know that "while atherosclerosis risk factors are associated with incident aortic stenosis, they are not

associated with the propagation phase of the disease and aortic stenosis progression. This is important when considering the development of novel aortic stenosis therapies that will need to slow disease progression rather than initiation if they are to be successful" (figure 3).

The study by Buoro and colleagues ${ }^{5}$ in this issue of Heart provides reference

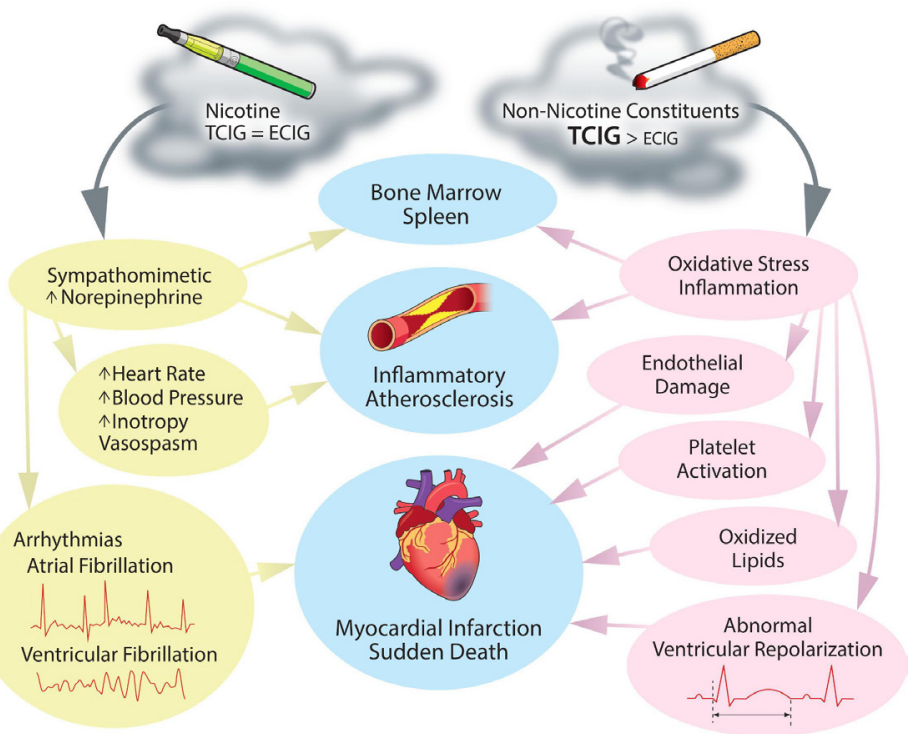

Figure 4 Cardiovascular effects of tobacco cigarettes (TCIGs) and electronic cigarettes (ECIGs). In considering the cardiovascular risks of tobacco cigarettes and electronic cigarettes, it is best to divide the risks into those largely attributable to nicotine and those largely attributable to nonnicotine constituents in their respective emissions. Cardiovascular effects associated with nicotine are attributable to nicotine's sympathomimetic effects, including increases in heart rate, blood pressure and inotropy, potentially accompanied by vasospasm, leading to ischaemia. Additionally, increased sympathetic tone is linked to heightened risk of arrhythmias, both atrial and ventricular. Increased sympathetic tone may be the instigator of activation of the splenocardiac axis, increasing inflammation, and once again increasing the risk of inflammatory atherosclerosis and ischaemia. ${ }^{25}$ Potential cardiovascular effects of non-nicotine constituents include increased oxidative stress and inflammation, leading to endothelial damage, platelet activation and lipid oxidation, increasing the risk of inflammatory atherosclerosis and ischaemia. Further, non-nicotine constituents may have adverse effects on ventricular repolarisation, once again increasing the risk of arrhythmias. values for normal pericardial fluid that will become a new clinical standard. This data shows that cell counts and levels of protein, albumin and lactate dehydrogenase that are considered inflammatory for other biological fluids are, in fact, normal for pericardial fluid. As Fender and $\mathrm{Zack}^{6}$ comment, "The findings of the study highlight the potential for diagnostic errors when interpretive tools are used outside of their validated purpose."

A review article in this issue of Heart by Shahandeh, Chowdhary and Middlekauff ${ }^{7}$ summarises the published evidence related to vaping and cardiac disease, including potential adverse physiological effects of electronic cigarettes (figure 4).

The Education in Heart article $^{8}$ in this issue reviews the diagnosis and management of cardiac sarcoidosis with useful clinical algorithms and examples of imaging findings.

Be sure to take a look at the short Cardiology-in-Focus article ${ }^{9}$ announcing the winner and finalists for the 2021 Heart Best Research Paper Award. Join me in congratulating these investigators on their dedication to the highest research standards and the value of their clinical research data.

Funding The authors have not declared a specific grant for this research from any funding agency in the public, commercial or not-for-profit sectors.

Competing interests None declared.

Patient and public involvement Patients and/or the public were not involved in the design, or conduct, or reporting, or dissemination plans of this research.

Patient consent for publication Not required.

Provenance and peer review Commissioned; internally peer reviewed.

(C) Author(s) (or their employer(s)) 2021. No commercial re-use. See rights and permissions. Published by BMJ.

\section{Check for updates}

To cite Otto CM. Heart 2021;107:1521-1523.

Heart 2021:107:1521-1523.

doi:10.1136/heartjnl-2021-320238

ORCID iD

Catherine M Otto http://orcid.org/0000-0002-05279392

\section{REFERENCES}

1 Tijssen A, Snaterse M, Minneboo M, et al. Weight management and determinants of weight change in patients with coronary artery disease. Heart 2021;107:1552-9.

2 Kelsey M, Pagidipati N. Towards a personalised approach for obesity treatment: one size does not fit all. Heart 2021;107:1526-7.

3 Khurrami L, Møller JE, Lindholt JS, et al. Cross-Sectional study of aortic valve calcification and cardiovascular risk factors in older Danish men. Heart 2021;107:1536-43. 
4 Williams MC, Dweck MR. Cardiovascular risk factors and aortic valve calcification: what do these associations mean? Heart 2021;107:1524-8.

5 Buoro S, Tombetti E, Ceriotti F. What is the normal composition of pericardial fluid? Heart 2021;107:1584-90.
6 Fender EA, Zack CJ. Shining a new light on pericardial fluid. Heart 2021;107:1528-9.

7 Shahandeh N, Chowdhary H, Middlekauff HR. Vaping and cardiac disease. Heart 2021;107:1530-5.
8 Kouranos V, Sharma R. Cardiac sarcoidosis: state-ofthe-art review. Heart 2021:107:1591-9.

9 Otto CM. Heart Best Research Paper Award 2021. Heart 2021;107:1600-7. 